\title{
Original
}

\section{Experimental Study on In Situ Tissue Engineering of the Temporomandibular Joint Disc using Autologous Bone Marrow and Collagen Sponge Scaffold}

\author{
Eizaburo Kobayashi, ${ }^{1,2)}$, Taka Nakahara ${ }^{2,3)}$, Masatoshi Inoue ${ }^{2)}$, Keiji Shigeno, \\ Akira Tanaka ${ }^{1)}$ and Tatsuo Nakamura ${ }^{2)}$ \\ 1) Department of Oral and Maxillofacial Surgery, School of Life Dentistry at Niigata, The Nippon Dental University, Niigata, Japan \\ 2) Department of Bioartificial Organs, Institute for Frontier Medical Sciences, Kyoto University, Kyoto, Japan \\ 3) Department of Developmental and Regenerative Dentistry, School of Life Dentistry at Tokyo, The Nippon Dental University, Tokyo, \\ Japan \\ (Accepted for publication, January 23, 2015)
}

\begin{abstract}
The temporomandibular joint (TMJ) disc plays an important role in mandibular motion. Once the TMJ disc perforates, spontaneous recovery does not occur. The aim of this study was to induce regeneration of the TMJ disc through an in situ tissue engineering method. Japanese white rabbits weighing $2.5-3.0 \mathrm{~kg}$ were used in this study. The TMJ was surgically exposed and a round full-thickness perforation of diameter $1.6 \mathrm{~mm}$ was made in the center of the disc. Animals were divided into A and B groups. In group A, a collagen sponge scaffold $(1.6 \mathrm{~mm}$ in diameter with a height of $1.0 \mathrm{~mm}$ ) was placed in the perforation. In group $\mathrm{B}$, a collagen sponge scaffold mixed with autologous bone marrow was placed in the perforation. In both groups, a control perforation was made on the opposite side and left unfilled. The TMJ discs with surrounding tissues were collected 2, 4 and 8 weeks after surgery and were subjected to histological evaluation. In group A, after 4 weeks the size of perforation was reduced, but after 8 weeks, the perforation had still not completely closed. In group $\mathrm{B}$, after 2 weeks, regeneration of the disc was seen and the site of the perforation was completely filled by new connective tissue. In the controls, after 8 weeks, the size of perforation was reduced, but it had not completely closed. The results indicate that collagen sponge scaffold with autologous bone marrow may have the potential to induce regeneration of a damaged TMJ disc.
\end{abstract}

Key words: Temporomandibular joint disc, Regeneration, Bone marrow, Collagen sponge scaffold, Rabbits

\section{Introduction}

The temporomandibular joint (TMJ) disc plays an important role in mandibular motion. As the TMJ disc moves forward and backward in harmony with the mandibular condyle, pressure to the temporal bone and the mandibular condyle will be dispersed. If excess pressure is applied to the TMJ continuously, the TMJ disc may sometimes get displaced. Clinically, disc perforation may occur during anterior disc displacement, and disc perforation leads to decreased TMJ function and finally to osteoarthrosis.

Once the TMJ disc perforates, spontaneous recovery does not occur. Therefore, several researchers tried to regenerate the TMJ disc. In the past reports about regeneration of the TMJ disc, Lang et al. ${ }^{1}$ prepared a perforation with a diameter of $1.6 \mathrm{~mm}$ in rabbit TMJ disc, Bosanquet et al. ${ }^{2}$ reported a perforation with a diameter

Correspondence to: Dr. Eizaburo Kobayashi, Department of Oral and Maxillofacial Surgery, School of Life Dentistry at Niigata, The Nippon Dental University, 1-8 Hamaura-cho, Chuuo-ku, Nigata city, Niigata, 9518580 Japan; Phone: +81-25-267-1500; Fax: +81-25-267-9061; E-mail: eizabu@ngt.ndu.ac.jp of $5 \mathrm{~mm}$ in sheep TMJ disc and Helmy et al. ${ }^{3)}$ created a perforation with a diameter of 4-6 mm in monkey TMJ disc. However, these perforations in TMJ discs were not completely closed and showed degenerative changes. According to Wallace et al. ${ }^{4}$, rabbit TMJ disc incision did not heal even if they were sutured after incision of the TMJ disc. Furthermore, Miyamoto et al. ${ }^{5)}$ observed perforation of dog TMJ discs over time; TMJ disc and temporal bone adhered together in 2 weeks, TMJ disc and temporal bone and mandibular condyle adhered together in 4 weeks, but TMJ disc perforation had not completely closed even by 12 weeks. These results indicate that when TMJ disc perforations are made in any animal, spontaneous regeneration does not occur, because of the lack of vascularity of TMJ disc area.

The bone marrow consists of several types of stem cell compartments, including hematopoietic stem cells, mesenchymal stem cells (MSCs), and endothelial progenitor cells. It is widely known that bone marrow-derived MSCs can differentiate to osteoblasts, chondrocytes, adipocytes, skeletal muscle cells, 
J.Hard Tissue Biology Vol.

ligament cord and tendon ${ }^{6}$. Liu et al. ${ }^{7}$ suggested that MSCs would serve as an effective treatment tool for joint restoration because MSCs can differentiate to osteoblasts or chondrocytes.

The purpose of this study was to induce regeneration of the TMJ disc using a combination of collagen sponge scaffold and autologous bone marrow and to evaluate it histologically.

\section{Materials and Methods}

\section{Animals}

A total of 18 male Japanese white rabbits weighing $2.5-3.0 \mathrm{~kg}$ were used in this study. During acclimatization for a fixed period, rabbits were allowed to drink water freely and they were fed pellets before and post surgery.

This study was approved by the Animal Experiment Committee of Institute for Frontier Medical Sciences, Kyoto University, Kyoto.

\section{Collagen sponge scaffold}

Atelopeptide collagen extracted from porcine skin by enzymatic treatment with pepsin (provided by Nippon Ham Foods Co.,Ltd, Japan) was used. This collagen consists of type I collagen (70-80 \%) and type III collagen (20-30\%), and has very low antigenicity due to removal of the telopeptides.

The collagen sponge scaffold was prepared as previously described by Nakahara et al. ${ }^{8,9)}$ Briefly, the $1 \%$ collagen solution was homogenized (Nissei AM-3, Nihonseiki Kaisha Ltd., Japan) at $8000 \mathrm{rpm}$ for 15 minutes, frozen at -80 degrees, and freezedried for 48 hours. Thermal crosslinking was done under pressure $\left(1 \times 10^{-1} \mathrm{~Pa}\right)$ at 140 degrees for 12 hours and the collagen sponge was prepared (Fig. 1).

\section{Surgical procedure}

The operation was performed under general anesthesia (pentobarbital sodium, Dainippon Sumitomo Pharma Co., Ltd, Japan) by intravenous injection into the auricular vein.

\section{Extraction of the autologous bone marrow}

A skin incision was made on the proximal edge of the thighbone and a needle was inserted in the bone. Approximately $1 \mathrm{ml}$ of bone marrow was aspirated.

\section{Surgical technique}

The TMJ was surgically exposed and a round full-thickness perforation of diameter $1.6 \mathrm{~mm}$ was made in the center of the disc using a fissure bur (Fig. 2).

\section{Experimental groups}

Animals were divided into two groups (A and B). In group A, a collagen sponge scaffold $(1.6 \mathrm{~mm}$ in diameter with a height of $1.0 \mathrm{~mm}$ ) was placed in the perforation (Fig. 3). In group B, a collagen sponge scaffold mixed with autologous bone marrow was placed in the perforation (Fig. 4). Six rabbits were used in each group.

\section{Control group}

In both groups, a control perforation was made on the opposite side and left unfilled. In the Sham operation group, the superior joint space was once surgically opened and closed immediately without making a perforation. A total of 6 rabbits were used in this group.

\section{Post-operative care}

For three days after the operation, ampicillin cloxacillin sodium (Viccillin ${ }^{\circledR}$, Meiji Seika Pharma Co., Ltd, Japan) $100 \mathrm{mg}$ was administered by intramuscular injection to prevent infection.

\section{Observation period}

Animals were sacrificed 2, 4 and 8 weeks after surgery and the TMJ was removed en bloc with surrounding tissues.

\section{Histological examination}

We performed histological examinations in each specimen using hematoxylin and eosin (HE) stain and toluidine blue (TB) stain in both groups.

\section{Results}

\section{Group $A(n=6)$}

No regeneration was observed in any of the specimens after 2 weeks (Fig. 5) and 4 weeks (Fig. 6). However, after 8 weeks, the size of perforation was reduced, but the perforation had still not completely closed (Fig. 7).

Group B ( $\mathrm{n}=6)$ :

After 2 weeks, regeneration of the disc was seen and the site of the perforation was completely filled by new connective tissue only in 1 specimen (Fig. 8). After 4 weeks, regeneration of the disc was seen and the site of the perforation was completely filled by new connective tissue in both specimens (Fig. 9). After 8 weeks, regeneration of the disc was seen and new connective tissue became thick in both specimens (Fig. 10).

Control group $(\mathrm{n}=6)$ :

No regeneration was observed in any of the specimens after 2 weeks (Fig. 11), 4 weeks (Fig. 12) and 8 weeks (Fig. 13).

\section{Discussion}

The TMJ joint consists of the mandibular condyle which is a part of the mandible, the articular eminence, the glenoid fossa, the articular disc and collagen fibers that exist between them. The TMJ exhibits not only rotary motion, but also gliding motion, compared with other joints which have only rotary motion. In other words, the mandibular condyle which lies in the glenoid 


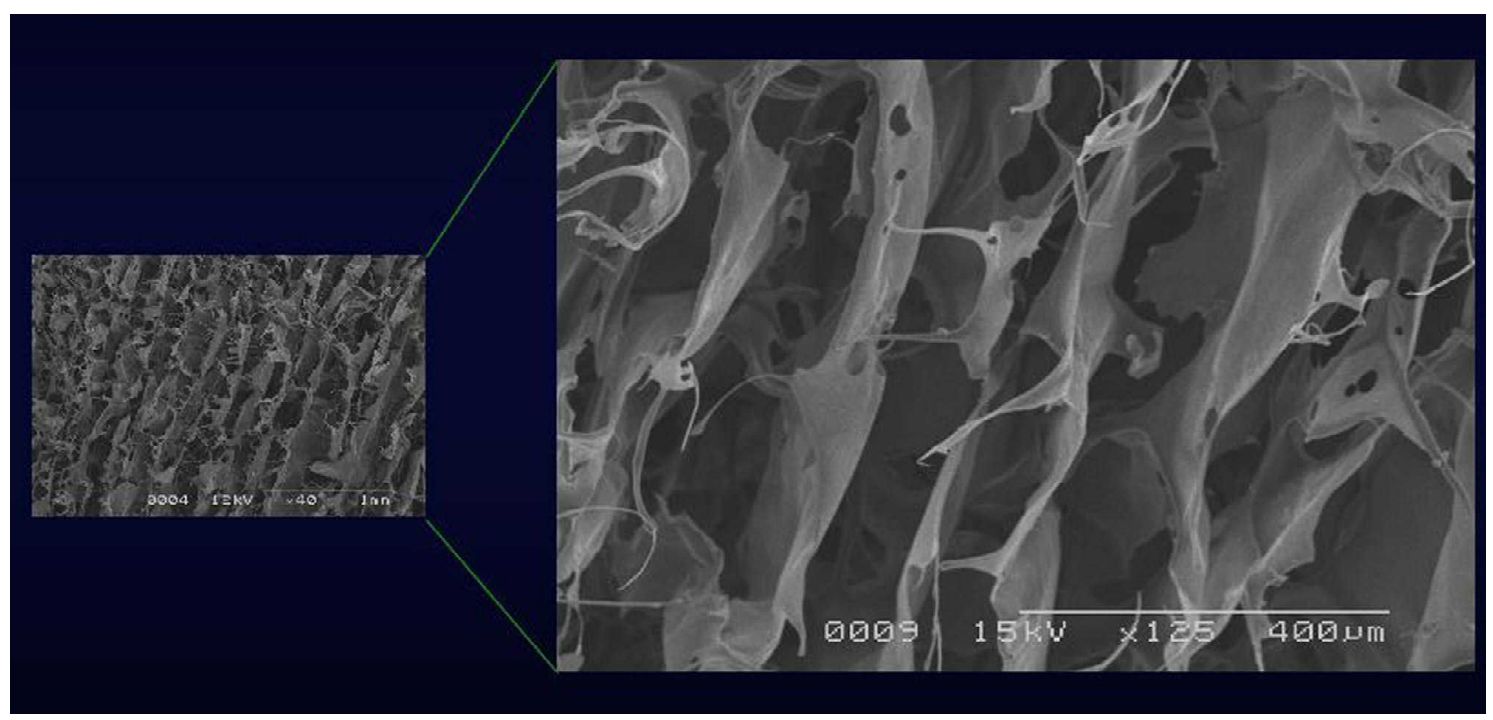

Figure 1. Scanning electron microscope image of collagen sponge.

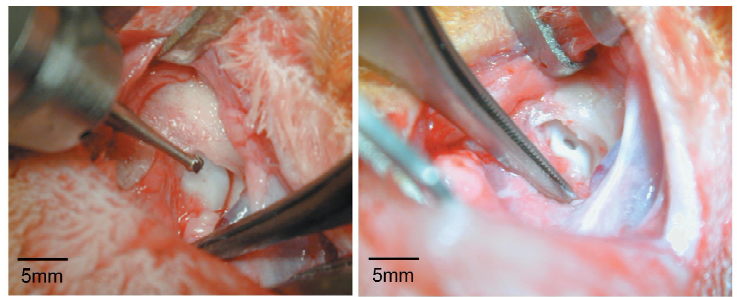

Figure 2. Perforation of diameter $1.6 \mathrm{~mm}$ was made in the center of the disc using a fissure bur.

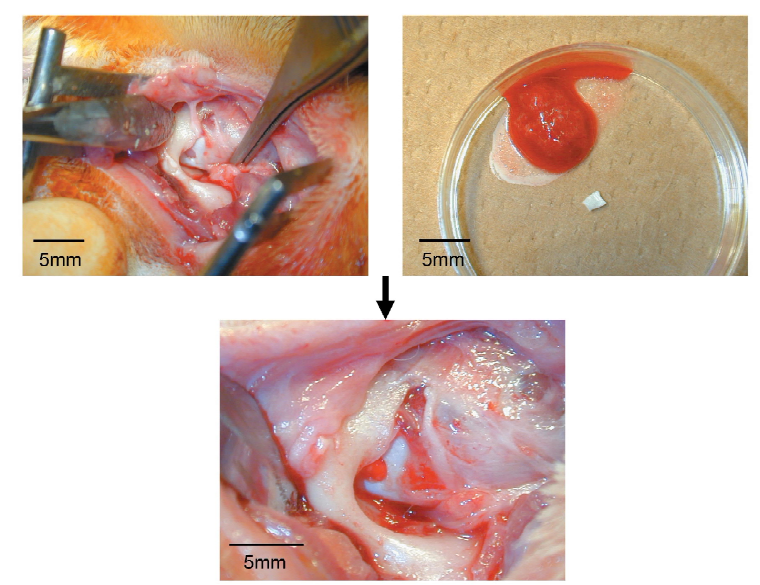

Figure 4. A collagen sponge mixed with autologous bone marrow was placed in the perforation in group B.

fossa at closing of the mouth glides during mouth opening and the articular disc enables smooth movement. Therefore, as the articular disc moves forward and backward in harmony with the mandibular condyle, pressure to the temporal bone and the mandibular condyle will be dispersed. If excess pressure is applied to the TMJ continuously, the articular disc may sometimes get displaced. However, the mechanism by which the TMJ disc

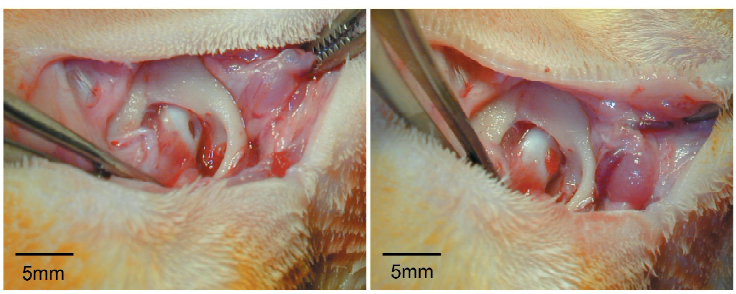

Figure 3. A collagen sponge $(1.6 \mathrm{~mm}$ in diameter with a height of 1.0 $\mathrm{mm})$ was placed in the perforation in group A.

displaces in an anterior direction has not been clarified.

According to the American Academy of Orofacial Pain (AAOP), TMJ disorders are classified into 1-5 types and 11 subtypes, including disk displacement classes.

The reason for disc perforation, which is the worst condition, is not clear, although Hanson et al. ${ }^{10)}$ stated that the disk will become thin because of changes in bone surface of mandibular condyle and temporal bone. Clinically, disc perforation may occur during anterior disc displacement. It is thought that once the TMJ disc perforates, spontaneous recovery does not occur, and leads to decreased TMJ function and finally osteoarthrosis.

Embryonic stem cells (ES cell) which are capable of differentiation and can increase to infinity were identified in 1998, and they were expected to make up for the limitations of stem cells. However, they were derived from destroyed embryo cells, restrictions have been placed on their preparation and application in various countries.

Yamanaka et al. ${ }^{11)}$ established human induced pluripotent stem cell (iPS cell) by artificially introducing four kinds of genes into a somatic cell in 2007. This iPS cell has multiple differentiation ability similar to ES cell and can increase to infinity. Moreover it solves the bioethics problem, so it is highly expected to become a potential cell source for new regenerative medicine. On the other 
J.Hard Tissue Biology Vol. 24(2):211 - 218, 2015
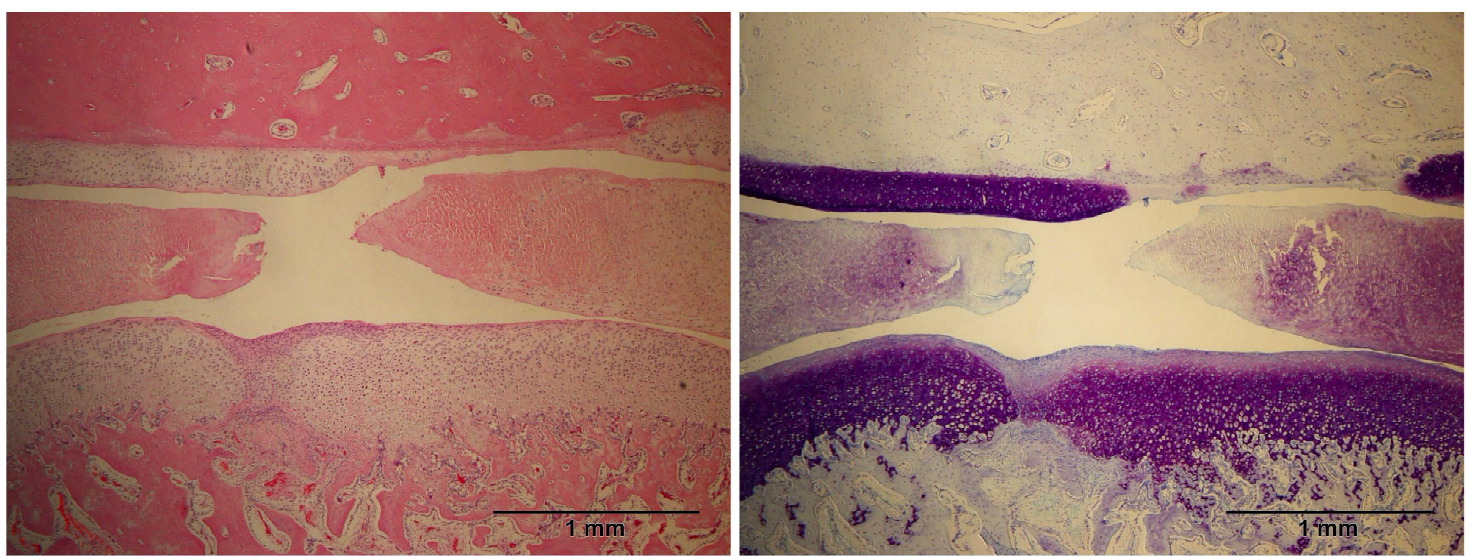

Figure 5. Group A after 2weeks, HE and TB.
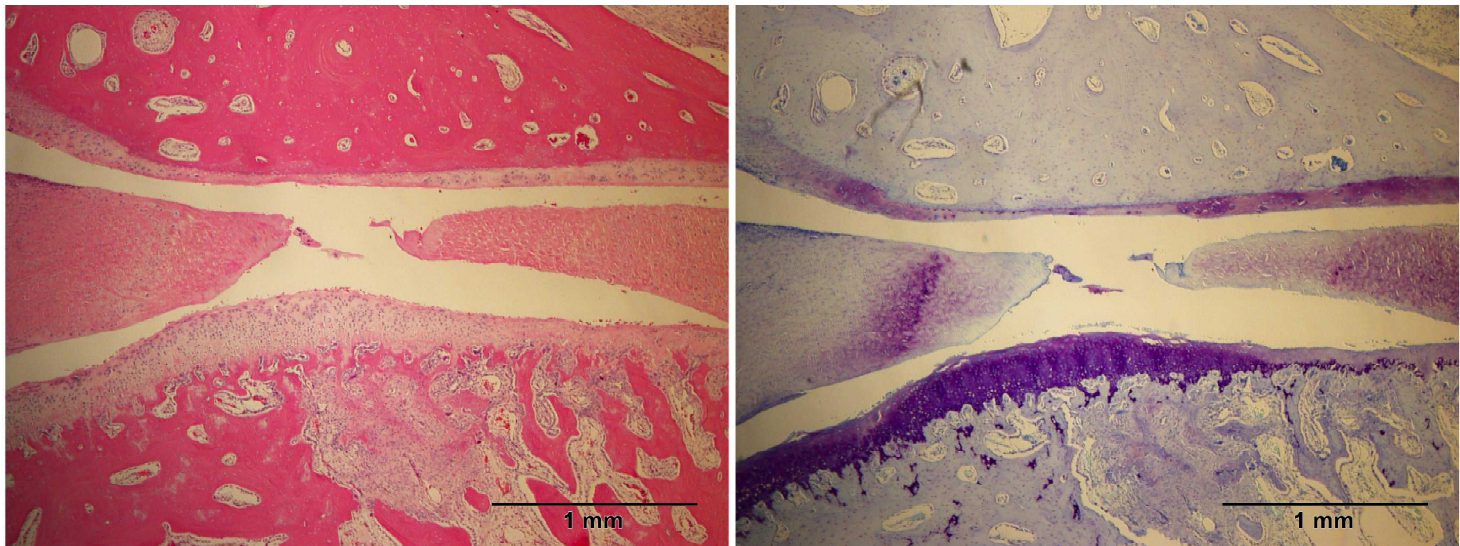

Figure 6. Group A after 4weeks, HE and TB.
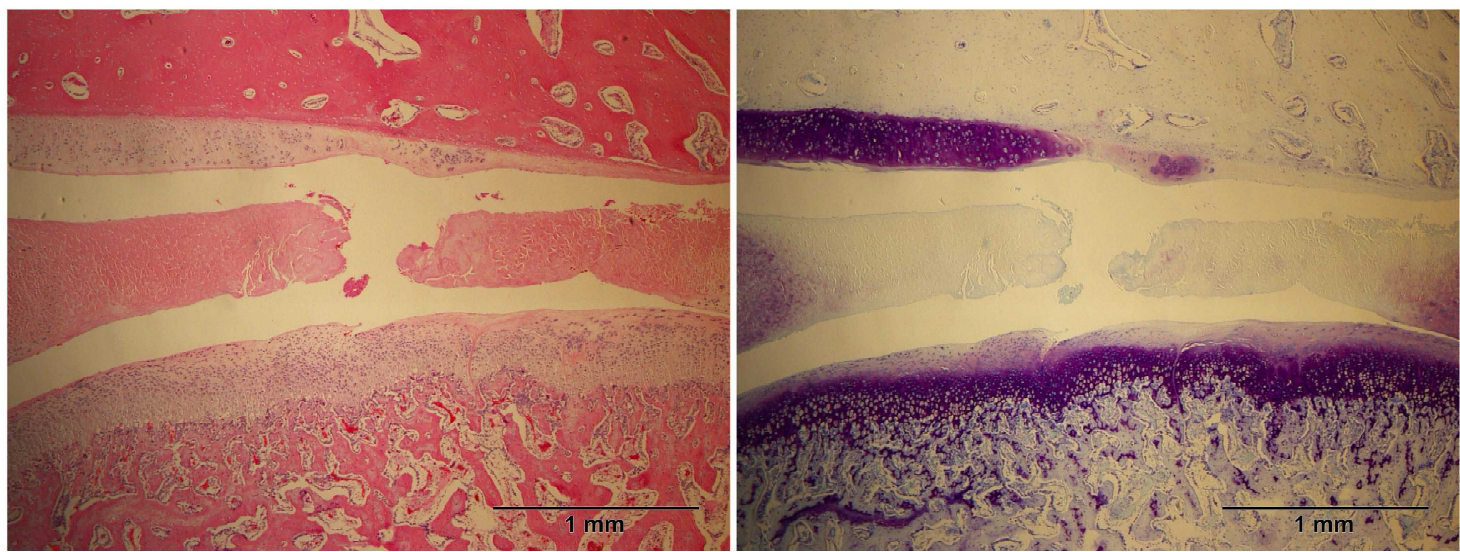

Figure 7. Group A after 8 weeks, HE and TB.

hand, there is the possibility of tumor occurrence or adverse immune reaction.

In recent years, MSCs were found in adult tissues, and they can differentiate into several cells ${ }^{12}$. As for mesodermal tissue, the stem cell is shown to exist in bone marrow, and research shows good progress.

The bone marrow cells consist of hemopoietic stem cells which differentiate into blood cells in the future, myeloblasts, erythroblasts, megakaryoblasts and bone marrow stromal cells which support hematopoietic cells. Marrow stromal cell secrete various growth factors which promote differentiation and proliferation of hematopoietic cells, and cytokines. According to a recent study ${ }^{13)}$, MSCs were included in the marrow stromal cell and they differentiate to various mesodermal cells. It is reported that MSCs can differentiate to osteoblasts, chondroblasts, adipocytes, skeletal muscle cells, ligament cord and tendon ${ }^{6}$. Furthermore, the MSCs have self-replication ability, and are present in cerebrospinal fluid, synovial membrane, periosteum, 
Eizaburo Kobayashi et. al.: In Situ Tissue Engineering of TMJ Disc
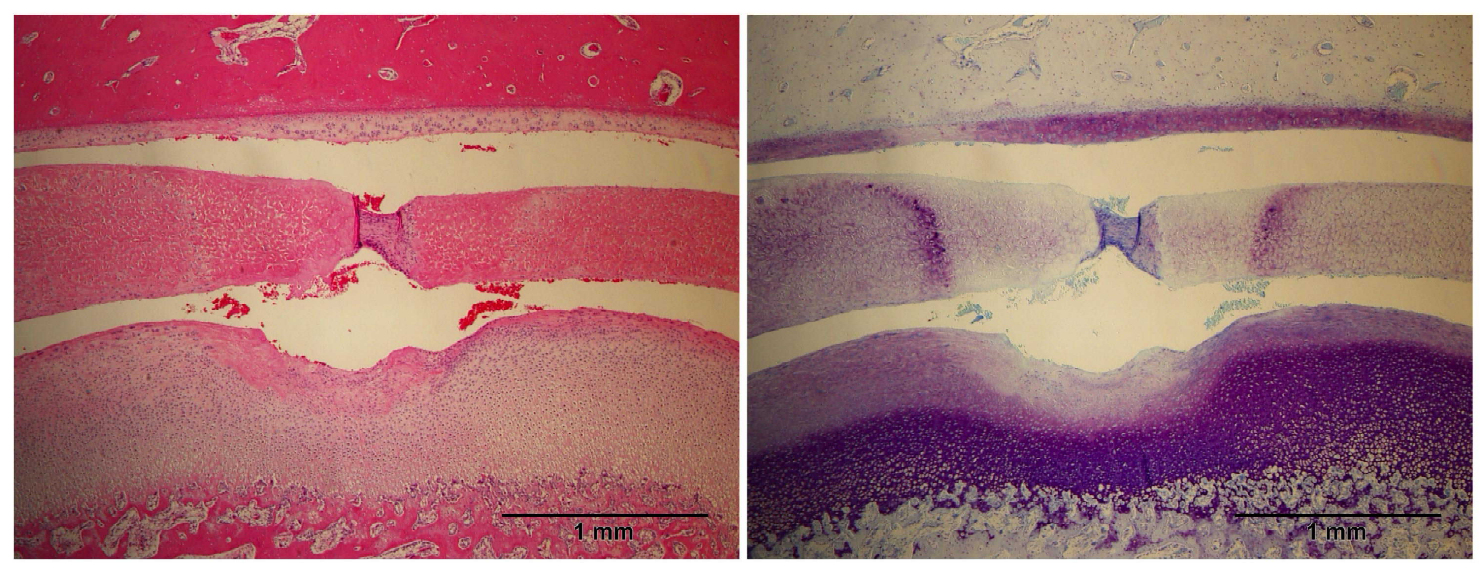

Figure 8. Group B after 2weeks, HE and TB.
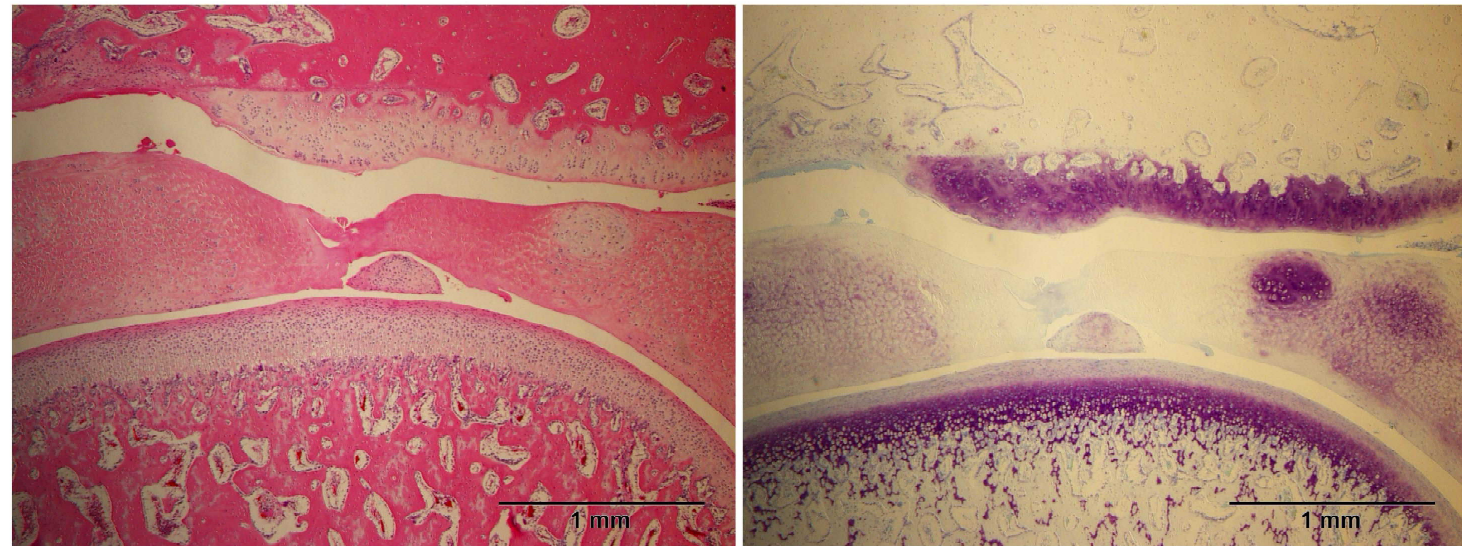

Figure 9. Group B after 4weeks, HE and TB.
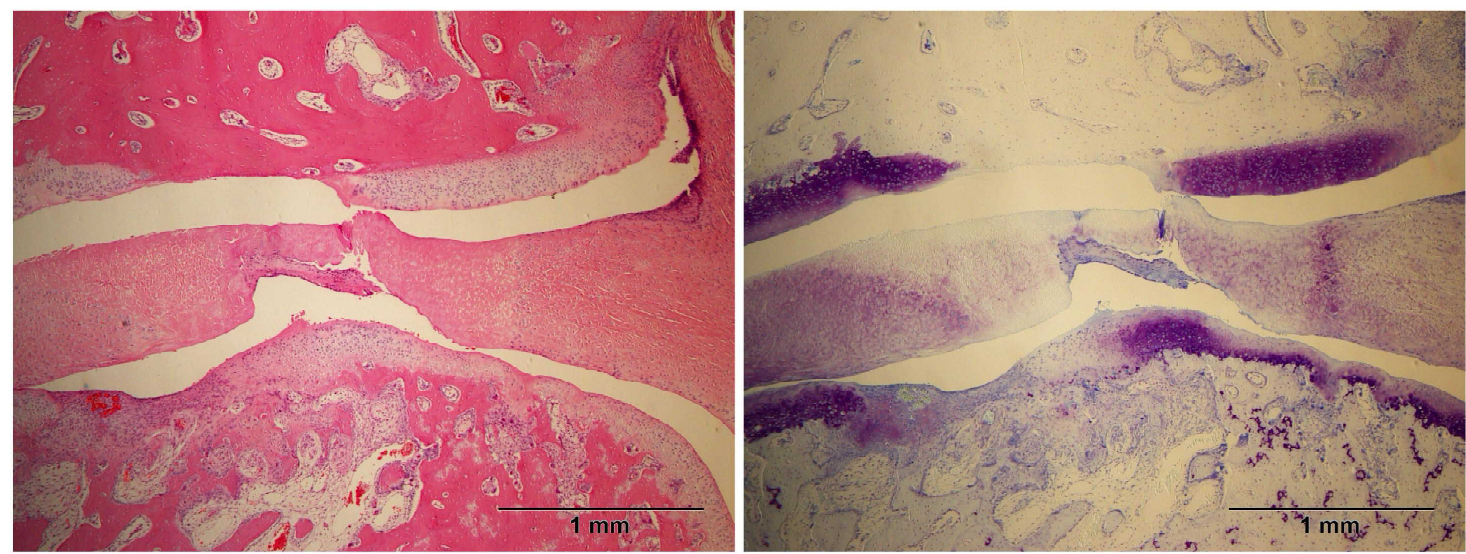

Figure 10. Group B after 8weeks, HE and TB.

fat, muscle cells, dental pulp, and periodontal ligament. Liu et al. ${ }^{7)}$ suggested that MSCs would serve as an effective treatment tool for joint restoration because MSCs can differentiate to osteoblasts or chondroblasts. Furthermore, MSCs do not have a bioethics problem and malignant transformation risk unlike ES cells or iPS cells.

In this experimental study, bone marrow was used as it was considered to be more practicable. The size of perforation was reduced by using collagen sponge alone, but it did not completely close even after 8 weeks. On the other hand, when using collagen mixed with autologous bone marrow, the perforation was replaced by new connective tissue after 2 weeks. This finding may indicate that collagen sponge with autologous bone marrow has the potential to induce regeneration of the damaged TMJ disc.

Recently, Jo et al. ${ }^{14)}$ reported that MSCs injection derived from adipocyte were useful in cartilage defects due to osteoarthritis in the knee joint. Quarch et al. ${ }^{15)}$ also transplanted autologous chondrocytes in 37 patients and obtained good results. Sakaguchi 
J.Hard Tissue Biology Vol. 24(2):211 - 218, 2015
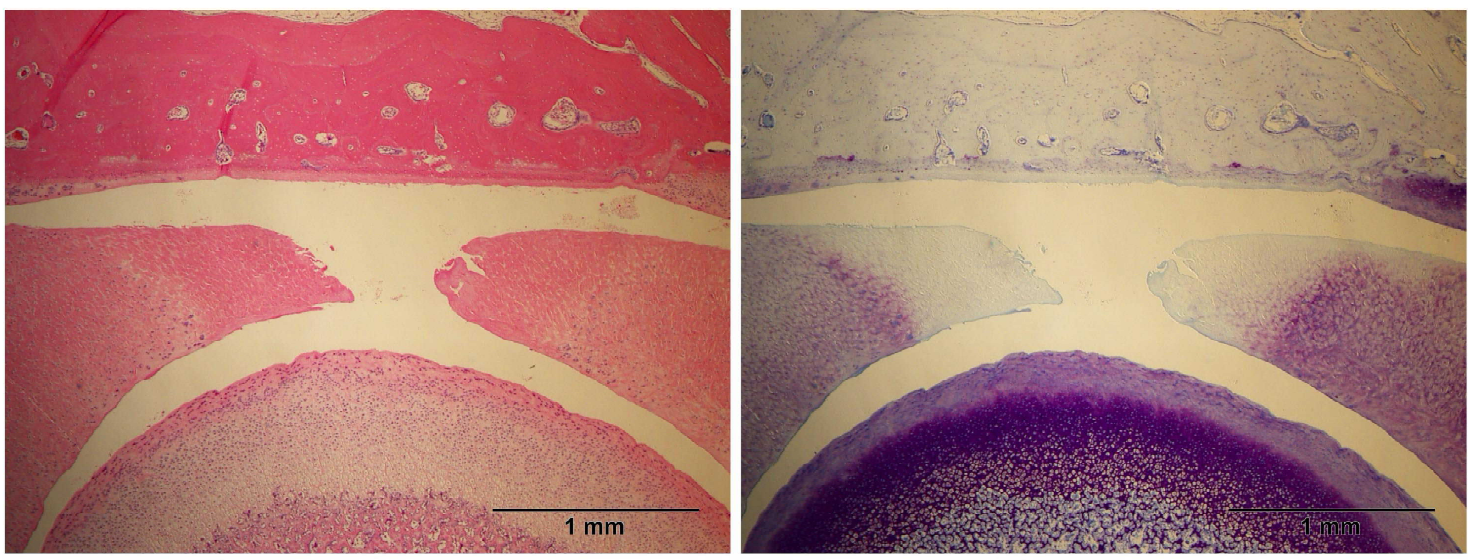

Figure 11. Control after 2weeks, HE and TB.
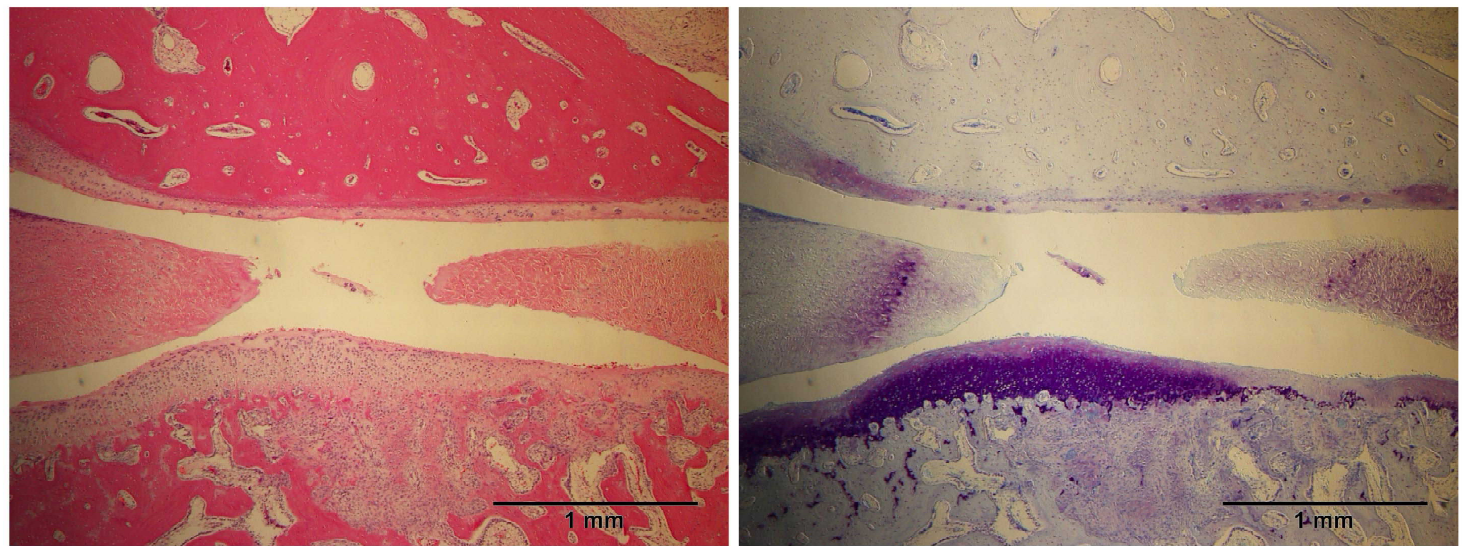

Figure 12. Control after 4weeks, HE and TB.
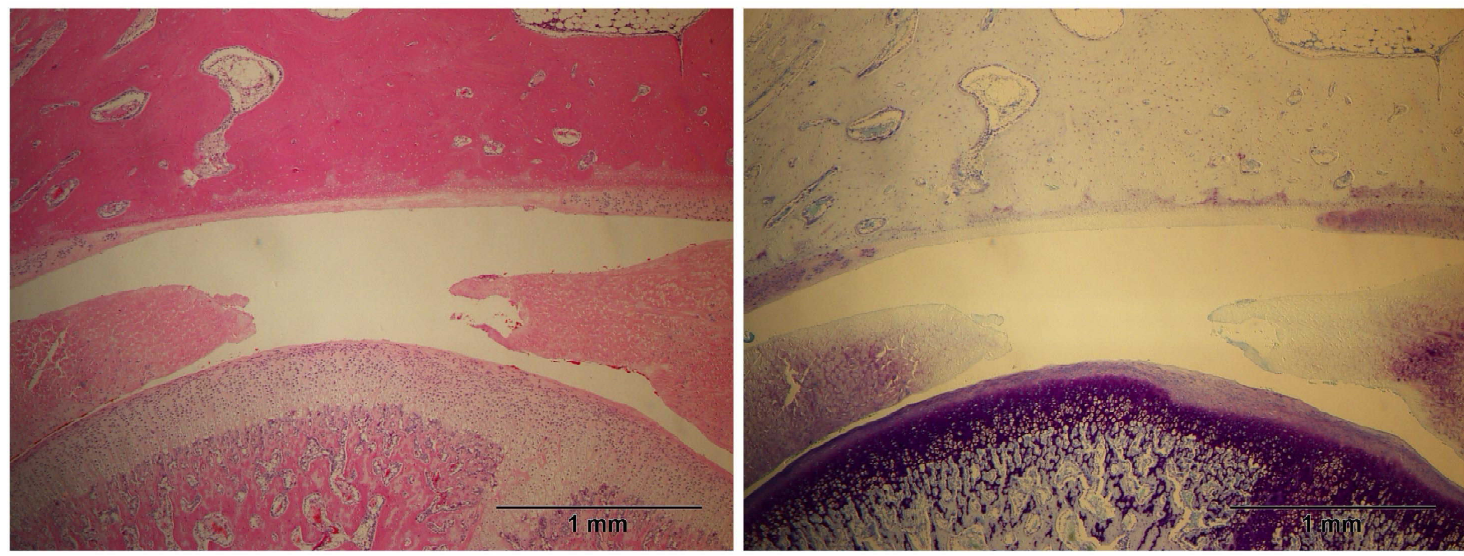

Figure 13. Control after 8weeks, HE and TB.

et al. ${ }^{16)}$ studied about comparison of the properties of human MSCs isolated from bone marrow, synovium, periosteum, skeletal muscle, and adipose tissue. They said that in chondrogenesis studies in which the cells were cultured from bone marrow, synovium, and periosteum were shown to be larger, and synoviumderived cells, in particular, had the greatest ability for chondrogenesis. Also, chondrocytes which were derived from adipocytes or muscle cells produced less cartilage matrix. It is thought that chondrocytes derived from bone marrow or synovial membrane would be more advantageous for cartilage production. In this study, bone marrow was used just as it is, and we hope to use chondrocytes selectively differentiated from bone marrow in the future. Furthermore, we plan to apply chondrocytes selectively differentiated from adipocytes which can be easily obtained from any part of the body.

This study has some limitations. Rabbit TMJ was used in this experiment. The TMJ in rabbit exhibits only rotary motion and no gliding motion, so the regeneration of TMJ disc when TMJ is 
Eizaburo Kobayashi et. al.: In Situ Tissue Engineering of TMJ Disc

in gliding motion needs to be further investigated. In dental field, regeneration experiments using MSCs are few and we hope that more experiments using MSCs will be conducted in the future.

\section{Acknowledgments}

The authors would like to thank the members of the Department of Bioartificial Organs, Institute for Frontier Medical Sciences, Kyoto University, Kyoto, Japan for their valuable help. This study was supported in part by a Grant-in-Aid for Scientific Research (A-2) from the Japan Society for the Promotion of Science (JSPS-13358016).

\section{References}

1. Lang TC, Zimny ML and Vijayagopal P. Experimental temporomandibular joint disc perforation in the rabbit: A gross morphologic, biochemical, and ultrastructural analysis. J Oral Maxillofac Surg 51: 1115-1128, 1993

2. Bosanquet A, Ishimaru J and Goss AN. Effect of experimental disc perforation in sheep temporomandibular joints. Int $\mathrm{J}$ Oral Maxillofac Surg 20: 177-181, 1991

3. Helmy E, Bays R and Sharawy M. Osteoarthrosis of the temporomandibular joint following experimental disc perforation in Macaca fascicularis. J Oral Maxillofac Surg 46: 979-990, 1988

4. Wallace DW and Laskin DM. Healing of surgical incisions in the disc and retrodiscal tissue of the rabbit temporomandibular joint. J Oral Maxillofac Surg 44: 965971, 1986

5. Miyamoto H, Shigematsu H, Hamao A, Magshi S, Suzuki S and Sakashita H. Effect of disc perforation on the temporomandibular joint: An experimental study and review of the literature on a canine model. Jpn J Oral Diag / Oral Med 16: 349-361, 2003

6. Bakhtina A, Tohfafarosh M, Lichtler A and Arinzeh TL. Characterization and differentiation potential of rabbit mesenchymal stem cells for translational regenerative medicine. In Vitro Cell Dev Biol Anim 50: 251-260, 2014

7. Liu N, Lyu X, Fan H, Shi J, Hu J and Luo E. Animal models for craniofacial reconstruction by stem/stromal cells. Curr Stem Cell Res Ther 9: 174-186, 2014
8. Nakahara T, Nakamura T, Kobayashi E, Inoue M, Shigeno K, Tabata Y, Eto K and Shimizu Y. Novel approach to regeneration of periodontal tissues based on in situ tissue engineering: Effects of controlled release of basic fibroblast growth factor from a sandwich membrane. Tissue Eng 9: 153-162, 2003

9. Nakahara T, Nakamura T, Kobayashi E, Kuremoto K, Matsuno T, Tabata Y, Eto K and Shimizu Y. In situ tissue engineering of periodontal tissues by seeding with periodontal ligament-derived cells. Tissue Eng 10: 537-544, 2004

10. Hansson $\mathrm{T}$ and Oberg T. Arthrosis and deviation in form in temporomandibular joint: A macroscopic study on human autopsy material. Acta Odontol Scand 35: 167-174, 1977

11. Takahashi K, Tanabe K, Ohnuki M, Narita M, Ichisaka T, Tomoda $\mathrm{K}$ and Yamanaka S. Induction of pluripotent stem cells from adult human fibroblasts by defined factors. Cell 131: 861-872, 2007

12. Mabuchi Y, Diarmaid D, Houlihan, Okano H and Matsuzaki Y. Discovering the true identity and function of mesenchymal stem cells. Inflamm Regen 32: 146-151, 2012

13. Muraoka R, Tsujigiwa H, Nakano K, Katase N, Tamamura R, Tomida M, Okafuji N, Nagatsuka H and Kawakami T. Transplanted bone marrow-derived cell migration into periodontal tissues and cell differentiation. J Hard Tissue Biol 20: 301-306, 2011

14. Jo CH, Lee YG, Shin WH, Kim H, Chai JW, Jeong EC, Kim JE, Shim H, Shin JS, Shin IS, Ra JC, Oh S and Yoon KS. Intra-articular injection of mesenchymal stem cells for the treatment of osteoarthritis of the knee: A proof-of-concept clinical trial. Stem Cells 32: 1254-1266, 2014

15. Quarch VM, Enderle E, Lotz J and Frosch KH. Fate of large donor site defects in osteochondral transfer procedures in the knee joint with and without TruFit Plugs. Arch Orthop Trauma Surg 134: 657-666, 2014

16. Sakaguchi Y, Sekiya I, Yagishita K, Muneta T. Comparison of human stem cells derived from various mesenchymal tissues: Superiority of synovium as a cell source. Arthritis Rheum. 52: 2521-2529, 2005 
J.Hard Tissue Biology Vol. 24(2):211 - 218, 2015 